\title{
Use of contrast-enhanced computed tomography in clinical staging of asymptomatic breast cancer patients to detect asymptomatic distant metastases
}

\author{
SATORU TANAKA, NAYUKO SATO, HIROYA FUJIOKA, YUKO TAKAHASHI, \\ KOSEI KIMURA, MITSUHIKO IWAMOTO and KAZUHISA UCHIYAMA \\ Section of Breast and Endocrine Surgery, Department of General and Gastroenterological Surgery, \\ Osaka Medical College, Osaka 569-8686, Japan
}

Received December 9, 2011; Accepted January 30, 2012

DOI: 10.3892/ol.2012.594

\begin{abstract}
The use of computed tomography (CT) with regards to the clinical staging of patients with asymptomatic breast cancer has been on the increase in clinical practice. However, the benefits of routine CT have yet to be fully clarified. This study investigated the value of employing contrast-enhanced CT (CECT) to screen for distant metastases in patients with asymptomatic breast cancer. The clinical records of 483 patients with asymptomatic breast cancer who underwent CECT at a single institution between April 2006 and January 2011 were reviewed retrospectively. The CECT results were classified into normal, true-positive (metastases) or false-positive findings. Abnormal CECT findings, including true- and falsepositive results, were detected in 65 patients (13.5\%). Of these, 26 patients (5.4\%) showed confirmed true metastatic disease, including 18 lung metastases, 11 liver metastases and 13 bone metastases. Upstaging to stage IV due to the results of the CECT scan occurred in 0 of 155 patients at stage I, 5 of 261 patients $(1.9 \%)$ at stage II and 21 of 67 patients $(31.3 \%)$ at stage III. The false-positive rates were 7.7, 9.0 and $8.7 \%$ in stages I, II and III, respectively. The size of the lung or liver metastasis was significantly larger than the false-positive lesion. Routine CECT did not appear to be useful for detecting distant metastases in completely asymptomatic patients. Conversely, a small number of patients were upstaged from early to stage IV and a predictive factor beyond $\mathrm{T}$ and $\mathrm{N}$ stage alone appears to be needed in order to predict which asymptomatic patients have distant metastases.
\end{abstract}

Correspondence to: Dr Satoru Tanaka, Section of Breast and Endocrine Surgery, Department of General and Gastroenterological Surgery, Osaka Medical College, 2-7 Daigaku-machi, Takatsuki City, Osaka 569-8686, Japan

E-mail: sur112@poh.osaka-med.ac.jp

Key words: breast cancer, computed tomography, distant metastasis, clinical staging

\section{Introduction}

The accurate clinical staging of patients with breast cancer is important in determining the most appropriate treatment. As the management of patients is significantly affected by the presence and extent of metastatic disease, the identification of unexpected distant metastases is likely to greatly impact the initial treatment strategy. Patients with distant metastases require systemic drug therapies rather than local treatments, including surgery. However, the vast majority of newly diagnosed patients do not exhibit symptoms or signs of metastatic disease. In addition, there is limited evidence to guide decisions on how patients should be staged.

At present, insufficient evidence supports the routine use of imaging to detect the presence of metastases in the initial assessment of patients with a small tumor and/or without lymph node metastases. Additonally, the National Comprehensive Cancer Network (NCCN) guidelines recommended the use of imaging only in cases with locally advanced disease or signs/symptoms that are suggestive of distant metastases (1). Notably, approximately $4 \%$ of breast cancer patients have detectable metastatic disease at the time of primary diagnosis and the majority of these patients exhibit symptoms of metastasis (2). However, if primary surgical treatment is performed after overlooking occult metastases in an asymptomatic patient, the delay in beginning systemic pharmacotherapy may affect the prognosis.

Conventionally, patients with suspected distant metastases are screened for occult metastases using plain chest radiography, liver ultrasonography and bone scintigraphy $(1,3)$, with computed tomography $(\mathrm{CT})$ used in certain patients $(4,5)$. Although the value of $\mathrm{CT}$ for asymptomatic patients and/or those with small tumors has not been clarified, its use in clinical staging is on the increase in clinical practice. The utility of contrast-enhanced CT (CECT) for diagnosing the intraductal tumor extension of breast cancer has been reported (6-9). The advantage of CECT is that tumor extension and distant metastases may be assessed in a single examination.

Previously, we reported the usefulness of CECT in the diagnosis of tumor extension (6). Routine CECT has also been performed in screening for distant metastases among patients 
with asymptomatic breast cancer. The present study investigated the value of staging CECT in detecting asymptomatic distant (lung, liver and bone) metastases in patients with primary breast cancer. Specifically, we aimed to determine how often occult metastases were detected and whether the prognosis of asymptomatic patients was affected.

\section{Materials and methods}

Patients. The results of 483 patients with asymptomatic breast cancer who underwent CECT at the Osaka Medical College Hospital (Osaka, Japan) between April 2006 and January 2011 were reviewed. Patients with previous cancer history, bilateral breast cancer or a diagnosis of in situ tumors were excluded from this study. All patients provided written informed consent for the CECT scan.

Scanning and classification of breast cancer. The CECT scans were performed using an Aquillion scanner (TOSHIBA Medical, Tokyo, Japan). The protocol in breast cancer patients is designed to cover the entire liver as well as the thoracic cavity. Following the plain CT scan, an enhanced zoomed scan was planned with a slice thickness of $0.5 \mathrm{~mm}$ and a pitch of $5.0 \mathrm{~mm}$. Non-ionic contrast medium $(100 \mathrm{ml} ; 300 \mathrm{mg} \mathrm{I} / \mathrm{g})$ was injected at a rate of $3 \mathrm{ml} / \mathrm{sec}$. A CECT scan from the thorax to the epigastrium was performed $210 \mathrm{sec}$ later, following bolus administration of the contrast medium and the breast scan. The CECT results were interpreted for the evidence of metastases by radiologists in our department.

Abnormal findings were classified as benign, metastatic lesion or indeterminate nodule. Indeterminate nodules required a follow-up CT scan (plain CT or CECT) within 3-4 months or further examinations, including magnetic resonance imaging (MRI) or positron emission tomography (PET) scans. Lesions that were unchanged on follow-up CT or that showed negative results on MRI or PET scans were classified as false-positive. All cases were then classified as normal, true-positive (metastatic disease) or false-positive. For the size of the lesions, the greatest dimensions in the findings of the CT were adopted. Staging was allocated according to the sixth edition of the tumor, node, metastasis (TNM) staging system of the American Joint Committee on Cancer (AJCC). The clinical assessment for $\mathrm{T}$ and $\mathrm{N}$ classifications were performed by physical examination and imaging modalities. The diagnosis of lymph node metastasis was judged on the basis of the size, laterality and internal structure of the lymph node.

Statistical analysis. Differences in the size of the primary breast tumor between the true-positive and normal/false-positive cases and the size of lesions detected by CECT in the true- and false-positive cases were evaluated using the non-parametric Mann-Whitney U test. The clinical factors and molecular markers associated with upstaging to stage IV by CECT were assessed using the Chi-square test or the Fisher's exact test when necessary due to the low frequency of individual cells. For the survival analysis among patients with normal, true- and false-positive findings, the Kaplan-Meier method and the logrank test were used. Data analysis was conducted using JMP ${ }^{\circledR}$ version 8.0.2 software (SAS Institute, Tokyo, Japan). $\mathrm{P}<0.05$ was considered to indicate a statistically significant result.
Table I. Descriptive characteristics of the 483 patients.

\begin{tabular}{|c|c|}
\hline Characteristic & $\mathrm{n}(\%)$ \\
\hline \multicolumn{2}{|l|}{ Age (years) } \\
\hline$<50$ & $108(22.4)$ \\
\hline$\geq 50$ & $375(77.6)$ \\
\hline \multicolumn{2}{|l|}{ T stage $(\mathrm{cm})$} \\
\hline $\mathrm{T} 1(\leq 2)$ & $161(33.3)$ \\
\hline $\mathrm{T} 2(>2-5.0)$ & $250(51.8)$ \\
\hline $\mathrm{T} 3(>5.0)$ & $43(8.9)$ \\
\hline T4 (chest wall or skin invasion) & $29(6.0)$ \\
\hline \multicolumn{2}{|l|}{$\mathrm{N}$ stage } \\
\hline No & $343(71.0)$ \\
\hline N1 & $115(23.8)$ \\
\hline $\mathrm{N} 2$ & $16(3.3)$ \\
\hline N3 & $9(1.9)$ \\
\hline \multicolumn{2}{|l|}{ Stage $^{a}$} \\
\hline $\mathrm{I}$ & $155(32.1)$ \\
\hline II & $261(54.0)$ \\
\hline III & $67(13.9)$ \\
\hline \multicolumn{2}{|l|}{ Histological grade } \\
\hline 1 & $146(30.2)$ \\
\hline 2 & $229(47.4)$ \\
\hline 3 & $50(10.4)$ \\
\hline Unknown $^{\mathrm{b}}$ & $58(12.0)$ \\
\hline \multicolumn{2}{|l|}{ Lymphovascular invasion } \\
\hline Yes & $153(31.7)$ \\
\hline No & $296(61.3)$ \\
\hline Unknown $^{\mathrm{c}}$ & $34(7.0)$ \\
\hline \multicolumn{2}{|l|}{ Estrogen receptor status } \\
\hline Positive & $381(78.9)$ \\
\hline Negative & $100(20.7)$ \\
\hline Unknown & $2(0.4)$ \\
\hline \multicolumn{2}{|l|}{ Progesterone receptor status } \\
\hline Positive & $314(65.0)$ \\
\hline Negative & $167(34.6)$ \\
\hline Unknown & $2(0.4)$ \\
\hline \multicolumn{2}{|l|}{ HER2 status } \\
\hline Positive & $65(13.5)$ \\
\hline Negative & 393 (81.4) \\
\hline Unknown & $25(5.2)$ \\
\hline
\end{tabular}

${ }^{\mathrm{a} A c c o r d i n g}$ to only $\mathrm{T}$ and $\mathrm{N}$ staging. ${ }^{\mathrm{b}} \mathrm{Of} 26$ patients with metastatic disease, 23 were unknown. ${ }^{\mathrm{c}}$ Of 26 patients with metastatic disease, 21 were unknown.

\section{Results}

The characteristics of the 483 patients are shown in Table I. According to $\mathrm{T}$ and $\mathrm{N}$ staging alone, 155 patients (32.1\%) were classified as stage I, $261(54.0 \%)$ as stage II and $67(13.9 \%)$ as stage III. Upstaging by CECT was significantly associated with larger tumor size [odds ratio, 33.4; 95\% confidence 
Table II. Clinicopathological factors associated with upstaging to stage IV by CECT.

\begin{tabular}{|c|c|c|c|}
\hline & \multicolumn{2}{|c|}{$\mathrm{n}(\%)$} & \multirow[b]{2}{*}{ P-value } \\
\hline & $\begin{array}{l}\text { Upstaged cases } \\
\text { (true-positive) }\end{array}$ & $\begin{array}{c}\text { Others } \\
\text { (normal/false-positive) }\end{array}$ & \\
\hline \multicolumn{4}{|l|}{ Age (years) } \\
\hline$<50$ & $8(30.8)$ & $101(22.1)$ & \multirow[t]{2}{*}{0.3} \\
\hline$\geq 50$ & $18(69.2)$ & $356(77.9)$ & \\
\hline Tumor size (median, $\mathrm{cm}$ ) & 6.1 & 2.5 & $<0.0001$ \\
\hline \multicolumn{4}{|l|}{ T stage } \\
\hline$\leq \mathrm{T} 2$ & $5(19.2)$ & $406(88.8)$ & \multirow[t]{2}{*}{$<0.0001$} \\
\hline$\geq \mathrm{T} 3$ & $21(80.8)$ & $51(11.2)$ & \\
\hline \multicolumn{4}{|l|}{$\mathrm{N}$ stage } \\
\hline$\leq \mathrm{N} 1$ & $13(50.0)$ & $445(97.4)$ & \multirow[t]{2}{*}{$<0.0001$} \\
\hline$\geq \mathrm{N} 2$ & $13(50.0)$ & $12(2.6)$ & \\
\hline \multicolumn{4}{|l|}{ Estrogen receptor status } \\
\hline Positive & $20(76.9)$ & $361(79.0)$ & \multirow[t]{3}{*}{0.62} \\
\hline Negative & $5(19.2)$ & $95(20.8)$ & \\
\hline Unknown & $1(3.8)$ & $1(0.2)$ & \\
\hline \multicolumn{4}{|l|}{ Progesterone receptor status } \\
\hline Positive & $15(57.7)$ & $299(65.4)$ & \multirow[t]{3}{*}{0.57} \\
\hline Negative & $10(38.5)$ & $157(34.4)$ & \\
\hline Unknown & $1(3.8)$ & $1(0.2)$ & \\
\hline \multicolumn{4}{|l|}{ HER2 status } \\
\hline Positive & $3(11.5)$ & $62(13.6)$ & \multirow[t]{3}{*}{0.55} \\
\hline Negative & $21(80.8)$ & $372(81.4)$ & \\
\hline Unknown & $2(7.7)$ & $23(5.0)$ & \\
\hline
\end{tabular}

CECT, contrast-enhanced computed tomograpy.

Table III. Detection of abnormal findings and distant metastases in each stage.

\begin{tabular}{|c|c|c|c|c|c|}
\hline Stage $^{a}$ & $\begin{array}{c}\text { No. of abnormal } \\
\text { lesions }(\%)\end{array}$ & $\begin{array}{l}\text { Lung metastases } \\
\qquad(\%)\end{array}$ & $\begin{array}{c}\text { Liver metastases } \\
(\%)\end{array}$ & $\begin{array}{c}\text { Bone metastases } \\
(\%)\end{array}$ & $\begin{array}{l}\text { Total no. of } \\
\text { lesions (\%) }\end{array}$ \\
\hline All $(n=483)$ & $65(13.5)$ & $18(3.7)$ & $11(2.3)$ & $13(2.7)$ & $26(5.4)$ \\
\hline $\mathrm{I}(\mathrm{n}=155)$ & $12(7.7)$ & $0(0)$ & $0(0)$ & $0(0)$ & $0(0)$ \\
\hline II $(n=261)$ & $28(10.7)$ & $2(0.8)$ & $1(0.4)$ & $4(1.5)$ & $5(1.9)^{\mathrm{b}}$ \\
\hline III $(n=67)$ & $25(37.3)$ & $16(23.9)$ & $10(14.9)$ & $9(13.4)$ & $21(31.3)^{\mathrm{c}}$ \\
\hline
\end{tabular}

${ }^{\mathrm{a} A c c o r d i n g}$ to $\mathrm{T}$ and $\mathrm{N}$ staging. ${ }^{\mathrm{b}}$ Two patients had only bone metastases. One patient had only lung metastasis. ${ }^{\mathrm{C}}$ Two patients had only bone metastases. Five patients had only lung metastases. Seven patients had lung and liver metastases.

interval (CI) 12.1-92.5; $\mathrm{P}<0.0001]$ and lymph node status (odds ratio, 37.1; 95\% CI 14.2-96.8; $\mathrm{P}<0.0001$; Table II). Overall, 65 patients $(13.3 \%)$ exhibited abnormal CECT findings, 12 of whom were classified as stage I, 28 as stage II and 25 as stage III (Table III). In total, 26 of 483 patients (5.4\%) were upstaged to stage IV by CECT, including 5 of 261 patients (1.9\%) in stage II and 21 of 67 patients $(31.3 \%)$ in stage III. Among the stage III patients who were upstaged to stage IV, $15(71.4 \%)$ were beyond stage IIIA, in contrast to 15 of 46
(32.6\%) non-upstaged patients who were beyond stage IIIA. Of the 26 upstaged patients, 18 had lung metastases, 11 had liver metastases and 13 had bone metastases (Table III). Of the 155 patients with stage I, none exhibitied true metastatic disease. Of the 261 stage II patients, only $2(0.8 \%)$ with lung metastases, one $(0.4 \%)$ with liver metastases and $4(1.5 \%)$ with bone metastases were detected. However, among the 67 stage III patients, 16 (23.9\%) with lung metastases, 10 (14.9\%) with liver metastases and $9(13.4 \%)$ with bone metastases were 
Table IV. Differences in size between true metastatic lesions and false-positive lesions.

\begin{tabular}{lcc}
\hline Type of lesion & Median size $(\mathrm{mm})$ & P-value \\
\hline Lung & 15 & \\
True metastasis & 5 & $<0.0001$ \\
False-positive & & \\
Liver & 20 & 0.004 \\
True metastasis & 8 & \\
False-positive & & \\
\hline
\end{tabular}

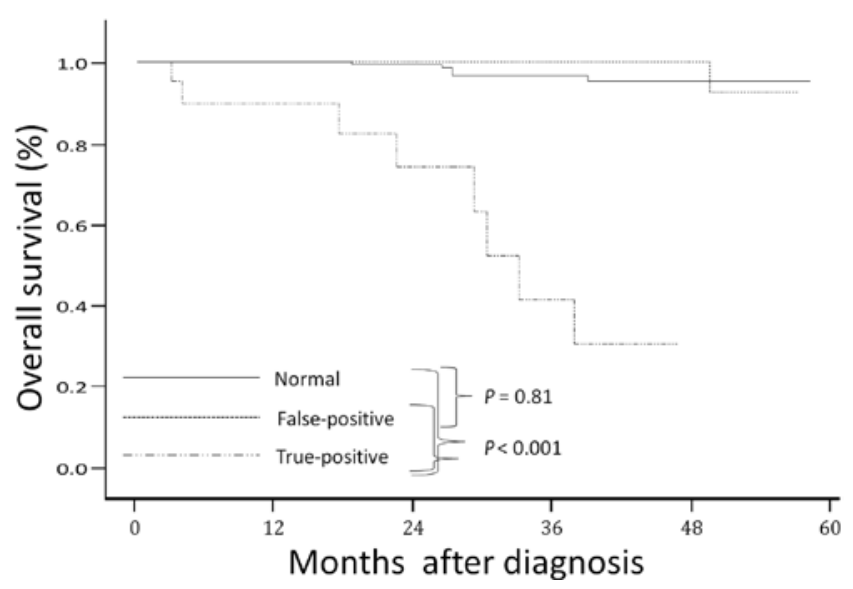

Figure 1. Kaplan-Meier estimates of the overall survival rate according to findings from contrast-enhanced computed tomography among normal, true-positive and false-positive cases.

detected. The false-positive rates for the total population $(8.5 \%)$ and according to each stage were comparable (7.7,9.0 and $8.7 \%$ in stages I, II and III, respectively). In the true-positive cases, the lung nodules and liver tumors were significantly larger than the potential lesions identified in false-positive cases (Table IV). The median size of lung metastasis was $15 \mathrm{~mm}$, compared with $5 \mathrm{~mm}$ in the false-positive cases $(\mathrm{P}<0.0001)$. The median size of liver metastasis was $20 \mathrm{~mm}$, compared with $8 \mathrm{~mm}$ in the false-positive cases $(\mathrm{P}=0.004)$.

According to the CECT findings, at a median follow-up period of 26 months (range, 1.0-60.1), significant differences in the overall survival rate were apparent among the normal, false-positive and true-positive cases (Fig. 1). The 2-year survival rate was $99 \%$ in patients with normal findings, $100 \%$ in patients with false-positive findings and $74 \%$ in patients with true-positive findings.

\section{Discussion}

Of the 483 patients with asymptomatic breast cancer investigated in the present study, the diagnosis of metastatic disease by CECT was useful for only 26 patients (5.4\%), identifying 18 cases of lung metastases, 11 liver metastases and 13 bone metastases. No patients were found to have metastatic disease in stage I, along with only 5 of 261 patients (1.9\%) with stage II. By contrast, 21 of 67 patients (31.3\%) with stage III were upstaged to stage IV and 15 patients $(71.4 \%)$ of those were originally stage IIIB or IIIC.

The presence of distant metastases in a patient with breast cancer upgrades the stage of disease, with concomitant prognostic and therapeutic implications. The location and extent of metastases are also of key importance, with the most common sites being the lung, liver and bone (10). In this study, only 4 patients showed metastases limited to bone. Unlike patients with only skeletal metastases, usually indicating a slow-growing disease, visceral involvement may progress rapidly and require aggressive systemic therapy, including chemotherapy or molecular-targeted therapy (11). No clear evidence supports the routine use of imaging techniques to detect metastases in patients with early-stage operable breast cancer (12-14) and NCCN guidelines suggest that staging investigations should only be considered in patients with disease beyond stage IIIA (T3, N1, M0) or in those who show symptoms thereof (1). In agreement with the results of the current study, the ability to detect metastatic lesions by plain CT was increased in asymptomatic patients with stage III disease (5). It should be noted that the patients with disease beyond stage IIIA accounted for almost $60 \%$ of all the cases that were upstaged to stage IV. Furthermore, two previous studies have revealed no benefit in either the survival or quality of life from the early detection of asymptomatic metastatic disease (15). However, CT has been shown to have distinct advantages in the identification of small visceral metastases compared with traditional chest plain radiography or liver ultrasonography (16-18) and 1.9 and $16.2 \%$ of patients with stage II and stage IIIA disease, respectively, were upstaged to stage IV in this study. In addition, when screening for visceral distant metastases, CECT offers a superior assessment of liver metastases (particularly small metastases) compared with plain CT. A liver tumor mistaken for metastasis, for example hemangioma, is more readily correctly diagnosed using CECT rather than plain CT. In this study, the positive predictive value for detecting liver metastases was higher $(47.8 \%)$ than that in another study that used plain CT screening (18.4\%) (5). Earlier detection and the aggressive systemic treatment of small metastatic disease may improve the survival of patients, due to advances in systemic therapies. Given the above results, the use of CT (whether plain or CECT) screening for patients with asymptomatic breast cancer may increase. However, to avoid the overuse of $\mathrm{CT}$, the clinical stages and the symptoms of patients, as well as other factors associated with tumor biology, including growth factor levels, are crucial in the prediction of the distant disease status in early breast cancer patients. The increased use of CT in breast cancer staging may result in increased medical costs and unnecessary exposure to ionizing irradiation, which has the potential to cause secondary carcinogenesis (4).

The current study has certain limitations that are to be considered. First, this study used retrospective data from one institute and the relatively small number of patients may have reduced the power of the conclusion. Few of the suspicious lesions identified in patients were confirmed histologically since the metastases and judgments of false-positive status were generally based on clinical assessments. Certain falsepositive results may have been true-positives according to histological findings. 
In conclusion, the routine use of CECT to screen for distant metastases in patients with asymptomatic primary breast cancer does not appear to be required, with the exception of patients with locally advanced disease. However, CECT as a technique is superior to that of plain $\mathrm{CT}$ in detecting liver metastases. Of note, since a few patients were upstaged to stage IV in our study, examinations should be conducted in all early-stage patients to ensure early systemic therapy is utilized. However, more studies are required to predict which asymptomatic patients may benefit from either a plain CT or CECT examination.

\section{References}

1. National Comprehensive Cancer Network (NCCN). NCCN clinical practice guidelines in Oncology v.2, 2011. http://www. nccn.org/professionals/physician_gls/pdf/breast.pdf. Accessed August 16, 2011.

2. Ravaioli A, Pasini G, Polselli A, et al: Staging of breast cancer: new recommended standard procedure. Breast Cancer Res Treat 72: 53-60, 2002

3. Puglisi F, Andreetta C, Fasola G, Cattaruzzi E and Geatti O: Bone scan for baseline staging in invasive breast cancer at the time of primary presentation. Breast Care 2: 358-364, 2007.

4. Barrett T, Bowden DJ, Greenberg DC, Brown CH, Wishart GC and Britton PD: Radiological staging in breast cancer: which asymptomatic patients to image and how. Br J Cancer 101: 1522-1528, 2009

5. Kim H, Han W, Moon HG, et al: The value of preoperative staging chest computed tomography to detect asymptomatic lung and liver metastasis in patients with primary breast carcinoma Breast Cancer Res Treat 126: 637-641, 2011.

6. Sumiyoshi K, Kani H, Nohara T, et al: A comparison study between multidetector-row $\mathrm{CT}$ and histopathological findings in terms of the extension diagnosis of breast cancer. J Jpn Surg Assoc 67: 1463-1472, 2006 (In Japanese with English abstract).
7. Prionas ND, Lindfors KK, Ray S, Huang SY, Beckett LA, Monsky WL and Boone JM: Contrast-enhanced dedicated breast CT: initial clinical experience. Radiology 256: 714-723, 2010.

8. Fujita T, Doihara H, Takabatake D, et al: Multidetector row computed tomography for diagnosing intraductal extension of breast carcinoma. J Surg Oncol 91: 10-16, 2005.

9. Akashi-Tanaka S, Fukutomi T, Sato N and Miyakawa K: The role of computed tomography in the selection of breast cancer treatment. Breast Cancer 10: 198-203, 2003.

10. Carbone PP, Jordan VC and Bonadonna G: Neoplasms of the breast. In: Medical Oncology. Calebresi P and Schein PS (eds). 2nd edition. McGraw Hill, New York, pp19-45, 1993.

11. Sherry MM, Greco FA, Johnson DH and Hainsworth JD: Metastatic breast cancer confined to the skeletal system: an indolent disease. Am J Med 81: 381-386, 1986.

12. Gerber B, Seitz E, Müller H, Krause A, Reimer T, Kundt G and Friese K: Perioperative screening for metastatic disease is not indicated in patients with primary breast cancer and no clinical signs of tumor spread. Breast Cancer Res Treat 82: 29-37, 2003.

13. Puglisi F, Follador A, Minisini AM, et al: Baseline staging tests after a new diagnosis of breast cancer: further evidence of their limited indications. Ann Oncol 16: 263-266, 2005.

14. Kasem AR, Desai A, Daniell S and Sinha P: Bone scan and liver ultrasound scan in the preoperative staging for primary breast cancer. Breast J 12: 544-548, 2006.

15. Hurria A, Leung D, Trainor K, Norton L and Hudis C: Screening chest imaging studies are not effective in the follow-up of breast cancer patients. J Oncol Manag 12: 13-15, 2003.

16. Mahner S, Schirrmacher S, Brenner W, Jenicke L, Habermann CR, Avril N and Dose-Schwarz J: Comparison between positron emission tomography using 2-[fluorine-18]fluoro-2-deoxy-Dglucose, conventional imaging and computed tomography for staging of breast cancer. Ann Oncol 19: 1249-1254, 2008.

17. Barrett T, Bowden DJ, Greenberg DC Brown CH, Wishart GC and Britton PD: Radiological staging in breast cancer: which asymptomatic patients to image and how. Br J Cancer 101: 1522-1528. 2009.

18. Coburn N, Przybysz R, Barbera L Hodgson D, Sharir S, Laupacis A and Law C: CT, MRI and ultrasound scanning rates: evaluation of cancer diagnosis, staging and surveillance in Ontario. J Surg Oncol 98: 490-499, 2008. 